OPEN ACCESS

Edited by:

William Cho,

Queen Elizabeth Hospital (QEH),

Hong Kong

Reviewed by:

Francesco Di Raimondo,

Università degli Studi di Catania, Italy

Francine Baumann,

University of New Caledonia, France

*Correspondence:

$\mathrm{Li} \mathrm{He}$

lihe126@hotmail.com

Fan Wang

fanndywang@foxmail.com

Specialty section:

This article was submitted to Molecular and Cellular Oncology,

a section of the journal

Frontiers in Oncology

Received: 25 September 2018 Accepted: 29 November 2018

Published: 08 January 2019

Citation:

Liu X-P, Yin X-H, Meng X-Y, Yan X-H, Wang $F$ and $H e L$ (2019) Development and Validation of a 9-Gene Prognostic

Signature in Patients With Multiple Myeloma. Front. Oncol. 8:615. doi: 10.3389/fonc.2018.00615

\section{Development and Validation of a 9-Gene Prognostic Signature in Patients With Multiple Myeloma}

\author{
Xiao-Ping Liu ${ }^{1}$, Xiao-Hong Yin ${ }^{1}$, Xiang-Yu Meng ${ }^{1}$, Xin-Hui Yan ${ }^{2}$, Fan Wang ${ }^{3 *}$ and $\mathrm{Li} \mathrm{He}^{4 *}$ \\ ${ }^{1}$ Zhongnan Hospital of Wuhan University, Wuhan, China, ${ }^{2}$ Department of Cardiology, the First Hospital of Lanzhou University, \\ Lanzhou, China, ${ }^{3}$ Department of Gastroenterology, Zhongnan Hospital of Wuhan University, Wuhan, China, ${ }^{4}$ Department of \\ Hematology, Zhongnan Hospital of Wuhan University, Wuhan, China
}

Background: Multiple myeloma (MM) is one of the most common types of hematological malignance, and the prognosis of MM patients remains poor.

Objective: To identify and validate a genetic prognostic signature in patients with MM.

Methods: Co-expression network was constructed to identify hub genes related with International Staging System (ISS) stage of MM. Functional analysis of hub genes was conducted. Univariate Cox proportional hazard regression analysis was conducted to identify genes correlated with the overall survival (OS) of MM patients. Least absolute shrinkage and selection operator (LASSO) penalized Cox proportional hazards regression model was used to minimize overfitting and construct a prognostic signature. The prognostic value of the signature was validated in the test set and an independent validation cohort.

Results: A total of 758 hub genes correlated with ISS stage of MM patients were identified, and these hub genes were mainly enriched in several GO terms and KEGG pathways involved in cell proliferation and immune response. Nine hub genes (HLA-DPB1, TOP2A, FABP5, CYP1B1, IGHM, FANCI, LYZ, HMGN5, and BEND6) with non-zero coefficients in the LASSO Cox regression model were used to build a 9-gene prognostic signature. Relapsed MM and ISS stage III MM was associated with high risk score calculated based on the signature. Patients in the 9-gene signature low risk group was significantly associated with better clinical outcome than those in the 9-gene signature high risk group in the training set, test, and validation set.

Conclusions: We developed a 9-gene prognostic signature that might be an independent prognostic factor in patients with $\mathrm{MM}$.

\footnotetext{
Keywords: multiple myeloma, weighted co-expression network analysis, prognostic signature, LASSO Cox proportional hazards regression model, survival
}

\section{INTRODUCTION}

Multiple myeloma (MM), originated from malignant plasma cells secreting monoclonal $\mathrm{M}$ protein, represents the second most common malignancy in hematological malignancies (1-3). MM is differentiated from monoclonal gammopathy of undetermined significance (MGUS) and smoldering multiple myeloma (SMM) by the presence of end-organ damage $(4,5)$. The clinical 
symptoms of $\mathrm{MM}$ range from asymptomatic forms to manifestations of anemia, bone pain, and eventually spontaneous of fractures, renal failure, and frequent infections (6-8). Thanks to the introduction of novel agents (proteasome inhibitors, immunomodulatory drugs, and monoclonal antibodies), the management strategies for MM have been improved considerably in the past decade (9-12). Accordingly, the clinical outcomes of patients with MM have been significantly improved, however, MM remains an incurable disease and the prognosis of patients with MM remains poor (with a median survival of approximately 3-4 years) (13).

The International Staging System (ISS) stage of MM, based on $\beta_{2}$-microglobulin $\left(\beta_{2} \mathrm{M}\right)$ and albumin (ALB), divides $M M$ patients into three different stages with significant dissimilar clinical outcomes. The ISS was a collaborative effort by investigators from 17 institutions worldwide and from data on 11,171 patients $(4,14)$. Patients with stage $1,2,3$ diseases have median survivals of $62,44,29$ months, respectively $(4,15)$.

Weighted gene co-expression network analysis (WGCNA), a systems biology algorithm that can be applied to describing the correlation patterns among genes across microarray samples, finding and summarizing modules of high related genes, and relating modules to certain clinical phenotype $(16,17)$, is widely used to facilitate the screening or identification of candidate biomarkers or therapeutic targets (18). Therefore, in the present study, we used WGCNA to screen potentially relevant molecular biomarkers correlated with the ISS stages of patients with MM. Moreover, we developed and validated the associated signature in patients with MM.

\section{METHODS}

\section{Microarray Data}

MM gene expression data and clinical information were downloaded from gene expression omnibus (GEO) database (https://www.ncbi.nlm.nih.gov/geo/) and ArrayExpress (http:// www.ebi.ac.uk/arrayexpress/). Affymetrix gene expression profiles were performed using Affymetrix Human Genome U133 Plus 2.0 (HG-U133 Plus_2.0) [GSE19784 (19) and GSE24080 (20)], Affymetrix Human Genome U133A Array(GSE6477) (21, 22), and Affymetrix GeneChip Human Gene 1.0 ST Array[E-MTAB-4032 (23)]. GSE19784, including 328 samples from patients with newly diagnosed MM, was used to construct the co-expression network. GSE24080, including 559 untreated MM samples, was randomly assigned patients in a 3:2 ratio to a training set and test set to develop and validate a prognostic signature. GSE6477, including 15 samples of normal donors, 73 samples of newly diagnosed MM, and 28 samples of relapsed $\mathrm{MM}$, was used to evaluate the risk score calculated based on the prognostic signature among normal donor, newly diagnosed MM, and relapsed MM. E-MTAB-4032 (including 151 untreated MM) was used as an independent validation cohort to evaluate the prognostic role of the signature. Raw data of GSE19784 and E-MTAB-4032 were preprocessed using the R/Bioconductor "affy" package (24) and oligo (25) package, respectively. Robust Multi-array Average (RMA) (26) normalized data of these two studies at gene level were analyzed. For GSE6477 and GSE24080, the raw data had been normalized using MAS5 method and the expression levels of genes were transformed using the logarithm function. Purified CD138+ plasma cells (including myeloma cells and normal plasma cells) in GSE19784, GSE6477, E-MTAB-4032, and GSE20480 were separated using positive magnetic cell sorting selection with CD138 magnetic microbeads and subjected to gene expression profiling (GEP) as mentioned previously (19-23).

\section{Construction of Co-expression Network and Identification of Hub Genes}

The R package "WGCNA" (27) was used to construct a coexpression network for genes with highest variances (top 10000) in GSE19784. Prior to constructing the co-expression network, we applied sample networks method which was introduced by Oldham et al to detect outliers (28). A sample was considered as outlying, if the associated Z.K value was <2.5. The soft threshold power $\beta$ was selected according to the scale-free topology criterion as introduced previously $(16,17)$. Subsequently, Pearson's correlations between each gene pair was calculated to generate a matrix of adjacencies, and then the adjacencies were transformed into topological overlap matrix (TOM) (29). Next, we conducted average linkage hierarchical clustering based on the TOM-based dissimilarity. The minimum module size and medium sensitivity was 30 and 2, respectively, and other parameters were default. After relating modules to the ISS stage of MM patients and calculating the associated Gene Significance (the correlation between the genes and the trait) and Module Membership (the correlation of the first principal component of the expression matrix of the corresponding module and the gene expression profile), we screened hub genes using a networkScreening function based on Gene Significance and Module Membership and genes with q.Weighetd value (qvalue (local FDR) calculated from weighted $p$-value of association with the ISS stage of MM) $<0.01$ were finally treated as hub genes (30).

\section{Functional Enrichment Analysis of Hub Genes}

To understand the biological function of the hub genes, we performed gene ontology (GO), and Kyoto Encyclopedia of Genes and Genomes (KEGG) enrichment analysis using the DAVID (31) online tool. GO and KEGG terms at $P<0.05$ and false discovery rate $(\mathrm{FDR})<0.05$ were considered significantly enriched and the significantly enriched GO and KEGG terms were visualized using R package "ggplot2" (32).

\section{Development of the Prognostic Signature Based on the Hub Genes}

To investigate the associations between the hub genes and the survival of MM patients, we performed univariate Cox proportional hazards regression model in GSE24080. Genes significantly correlating with the overall survival (OS) of MM patients were included in a Least absolute shrinkage and selection operator (LASSO) penalized Cox proportional hazards regression model to minimize overfitting, and a 10 -fold cross 
validation was also conducted using the $\mathrm{R}$ package glmnet (33, 34). Then, we calculated the risk score for each patient based on this penalized Cox proportion model in the training set.

\section{Validation of the Predictive Value of the Prognostic Signature in MM Patients}

To validate the predictive value of the prognostic signature, Kaplan-Meier survival analysis, and univariate and multivariable Cox proportional hazards regression model were performed in the training set and test set in terms of OS, and event-free survival (EFS). Prior to multivariable Cox proportional hazards regression analysis on the OS, and EFS, we performed a variable selection based on the LASSO penalized Cox proportional hazards regression model. The definitions of OS and EFS was introduced previously (21-23). Meanwhile, we also validated the performance of the signature in the independent cohort EMTAB-4032. The above survival analyses were conducted using the R packages "survival" (35) and "survminer" (version 0.4.3). MM patients in GSE24080 and E-MTAB-4032 were classified into the prognostic low risk group and the 9-signature high risk group based on the cutoff calculated through time dependent receiver operating characteristic (ROC) analysis using the $\mathrm{R}$ package "survivalROC" (36). The risk score of the signature in patients with ISS I, II, and III disease were evaluated using E-MTAB-4032. Meanwhile, the risk score of the signature in normal plasma cells, untreated MM, and relapsed MM were evaluated using GSE6477. The risk scores of the signature in E-MTAB-4032 and GSE6477 were presented as mean \pm the standard error of the mean (SEM). Grouped data was analyzed using unpaired $T$-test, and $P<0.05$ was considered statistically significant.

\section{RESULTS}

\section{Results of Data Preprocessing, Co-expression Network Construction and Hub Genes Identification}

No sample was demonstrated to be an outlier after all samples were clustered based on their Euclidean distances. Meanwhile,
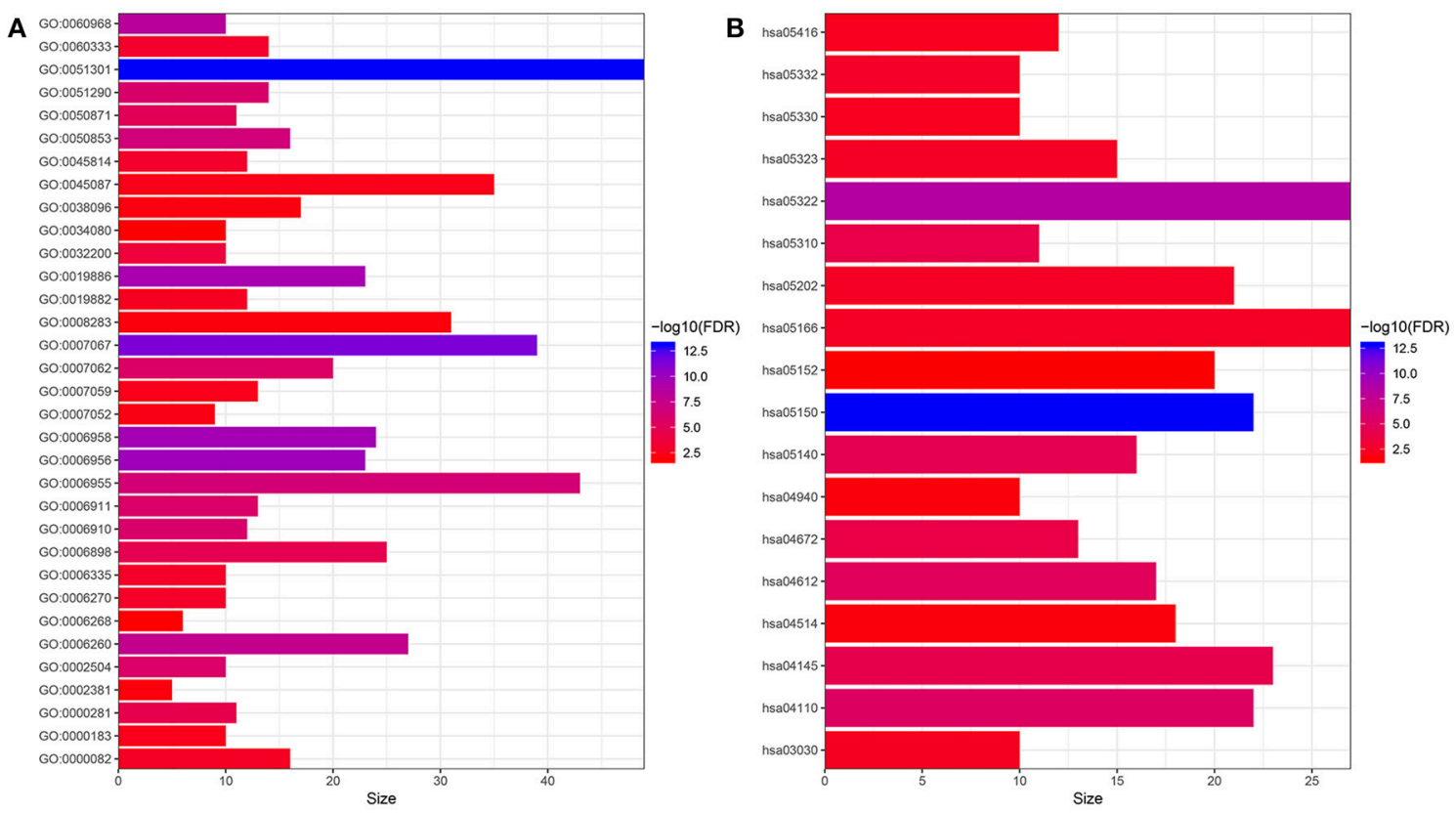

FIGURE 1 | Functional enrichment analysis of hub genes. (A) GO enrichment analysis. (B) KEGG enrichment analysis. GO:0051301 cell division, GO:0007067 mitotic nuclear division, GO:0006956 complement activation, GO:0006958 complement activation, classical pathway, GO:0019886 antigen processing and presentation of exogenous peptide antigen via MHC class II, GO:0060968 regulation of gene silencing, GO:0006260 DNA replication, GO:0050853 B cell receptor signaling pathway, GO:0006955 immune response, GO:0051290 protein heterotetramerization, GO:0006910 phagocytosis, recognition, GO:0006911 phagocytosis, engulfment, GO:0002504 antigen processing and presentation of peptide or polysaccharide antigen via MHC class II, GO:0007062 sister chromatid cohesion, GO:0050871 positive regulation of B cell activation, G0:0006898 receptor-mediated endocytosis, GO:0000281 mitotic cytokinesis, GO:0032200 telomere organization, GO:0060333 interferon-gamma-mediated signaling pathway, GO:0045814 negative regulation of gene expression, epigenetic, GO:0006335 DNA replication-dependent nucleosome assembly, GO:0006270 DNA replication initiation, GO:0000082 G1/S transition of mitotic cell cycle, GO:0019882 antigen processing and presentation, GO:0007059 chromosome segregation, GO:0000183 chromatin silencing at rDNA, GO:0045087 innate immune response, GO:0007052 mitotic spindle organization, G0:0038096 Fc-gamma receptor signaling pathway involved in phagocytosis, GO:0008283 cell proliferation, GO:0002381 immunoglobulin production involved in immunoglobulin mediated immune response, GO:0006268 DNA unwinding involved in DNA replication, GO:0034080 CENP-A containing nucleosome assembly; hsa05150:Staphylococcus aureus infection, hsa05322:Systemic lupus erythematosus, hsa04110:Cell cycle, hsa04612:Antigen processing and presentation, hsa05140:Leishmaniasis, hsa04145:Phagosome, hsa05310:Asthma, hsa04672:Intestinal immune network for IgA production, hsa05332:Graft-vs.-host disease, hsa05166:HTLV-I infection, hsa05202:Transcriptional misregulation in cancer, hsa05323:Rheumatoid arthritis, hsa03030:DNA replication, hsa05330:Allograft rejection, hsa05416:Viral myocarditis, hsa04940:Type I diabetes mellitus, hsa04514:Cell adhesion molecules (CAMs), hsa05152:Tuberculosis. 
$\beta=12$, the lowest power for which the scale-free topology fit index reaches 0.9 , was used for the subsequent adjacency calculation. After TOM based clustering, 14 gene modules were obtained. After co-expression network construction, a total of 780 probes were identified based on our screening criteria, 758 of which annotated to gene symbol were treated as hub genes (Supplementary Table 1). The major process of co-expression network construction and hub gene identification was shown in Supplementary Figure 1.

\section{GO and KEGG Pathway Enrichment Analysis of Hub Genes}

In order to have a preliminary understanding of the biological significance, we conducted GO and KEGG enrichment analysis. As shown in Figure 1, the hub genes were mostly enriched in GO terms related to cell proliferation ("cell division," "cell proliferation," "mitotic nuclear division," "DNA replication," "DNA unwinding involved in DNA replication," "sister chromatid cohesion," "mitotic cytokinesis," "DNA replication-dependent nucleosome assembly," "DNA replication initiation," "G1/S transition of mitotic cell cycle," "chromosome segregation," and "mitotic spindle organization") and immune response ("complement activation," "antigen processing and presentation of exogenous peptide antigen via MHC class II," “ B cell receptor signaling pathway," “ immune response," "phagocytosis, recognition," " positive regulation of B cell activation," "receptor-mediated endocytosis," "interferon-gamma-mediated signaling pathway," "innate immune response," "Fc-gamma receptor signaling pathway involved in phagocytosis," and "immunoglobulin production involved in immunoglobulin mediated immune response") (Figure 1A). Furthermore, the results of KEGG pathway enrichment analysis of the hub genes suggested that these genes were mainly enriched in infection or immune related pathways ("Staphylococcus aureus infection," "antigen processing and presentation," "leishmaniasis," "asthma," "intestinal immune network for IgA production," "graft-vs.host disease," "HTLV-I infection,"and "Rheumatoid arthritis"), and cell proliferation ("cell cycle,"and "DNA replication") (Figure 1B).

\section{Development of a 9-Gene Signature in Patients With MM}

To investigate the prognostic value of the hub genes, we conducted univariated Cox proportional hazards regression analysis, and the results suggested that the expression of $325 \mathrm{hub}$ genes were significantly correlated with the OS of MM patients in the training set of GSE24080. To avoid overfitting as much as possible, we conducted LASSO penalized Cox proportional hazards regression model in the training set in GSE24080, and the results identified 9 of the 325 hub genes (HLADPB1(major histocompatibility complex, class II, DP beta 1), TOP2A (topoisomerase 2A), FABP5 (Fatty Acid-Binding Protein 5), CYP1B1(cytochrome P450 family 1 subfamily B member 1), IGHM (immunoglobulin heavy constant mu), FANCI (FA complementation group I), LYZ (lysozyme), HMGN5 (high mobility group protein N5 subtype), and BEND6 (BEN domain containing 6) with non-zero coefficient. Thus, we built the 9-gene signature on the basis of the coefficients of these 9 hub genes in the LASSO penalized model (Supplementary Table 2).

\section{Associations Between the 9-Gene Signature and the ISS Stage of MM and the Disease Status}

Firstly, we calculated the risk score of each MM patients for the 9-gene signature in GSE6477 and E-MTAB-4032 based on the coefficients of the 9 hub genes (Supplementary Table 2). As shown in Figure 2A, the risk score of patients with ISS stage III disease was significantly higher than that of patients with ISS stageI $(t=-0.362, P=0.001)$ and II $(t=-0.218$, $P=0.031)$. Meanwhile, the risk score of patients with relapsed MM was significantly higher compared with that of normal donor $(t=5.782, P<0.001)$ and patients with untreated MM ( $t=2.977, P=0.004)$, and the risk score were higher in patients with untreated MM compared with that in normal donor $(t=-4.13, P<0.001$, Figure 2B).

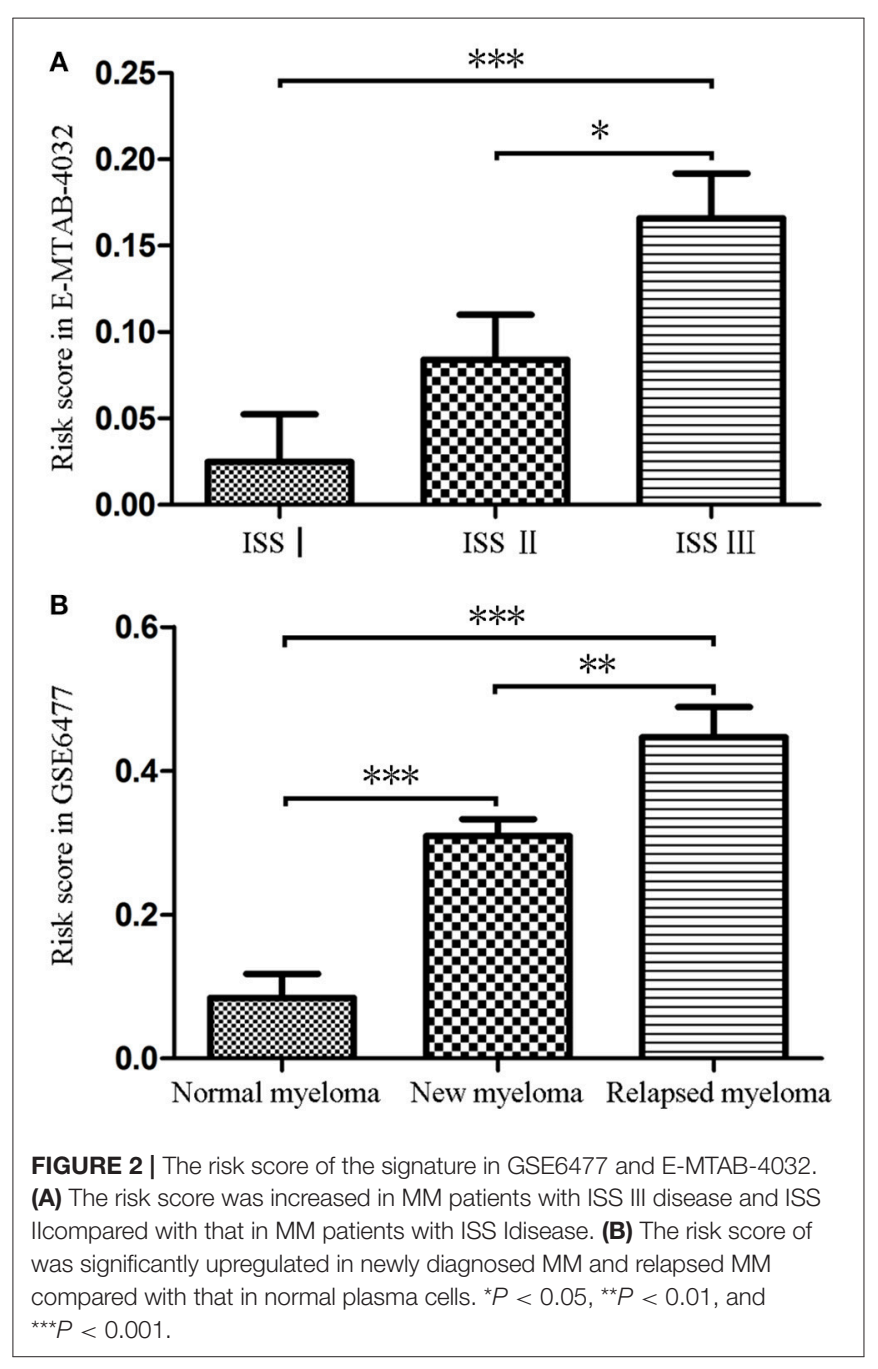




\section{Evaluation the Prognostic Role of the 9-Gene Signature}

The predictive value of the 9-gene signature was evaluated in the training set, test set and an independent set E-MTAB4032. Based on the optimal cutoff (1.939) calculated using time dependent ROC analysis (Supplementary Figure 1). The Kaplan-Meier survival analysis suggested that patients in the 9-gene signature low risk group had better OS compared with those in the 9-gene signature high risk group in the training set $(\mathrm{HR}=0.2664,95 \% \mathrm{CI}$ : 0.1772-0.4007, log-rank $P<0.001$, Figure 3A, Supplementary Table 3) and the test set $(\mathrm{HR}=0.5115,95 \% \mathrm{CI}: 0.3137-0.8339, \log -\operatorname{rank} P=0.0062$, Figure 3B, Supplementary Table 4). Meanwhile, patients in the 9-gene signature low risk group had with better EFS compared with those in the 9-gene signature high risk group in the training set $(\mathrm{HR}=0.3321,95 \%$ CI:0.2395-0.4606,
$P<0.0001$, Figure 3C, Supplementary Table 5) and test set $(\mathrm{HR}=0.5174,95 \% \mathrm{CI}: 0.3447-0.7765, \quad P=0.0015$, Figure 3D, Supplementary Table 6). Based on the results of variable selection (Supplementary Table 7), age, B2M ( $\beta 2$-microglobin), CRP (C reaction protein), LDH (lactate dehydrogenase), BMPC (bone marrow plasma cell), MRI (magnetic resonance imaging), and the 9-gene signature was included multivariable Cox proportional hazards regression analysis which indicated that the 9-gene signature was an independent prognostic factor in terms of OS and EFS in the training set and test (Supplementary Tables 3-6). Moreover, MM patients in the 9-gene low risk group also had better OS compared with those in the 9-gene signature high risk group in the independent validation cohort EMTAB-4032 (HR = 10.6091, 95\% CI: 3.2120-35.0409, log-rank $P=0.0061$, Figure 4). Meanwhile, the results of multivariable
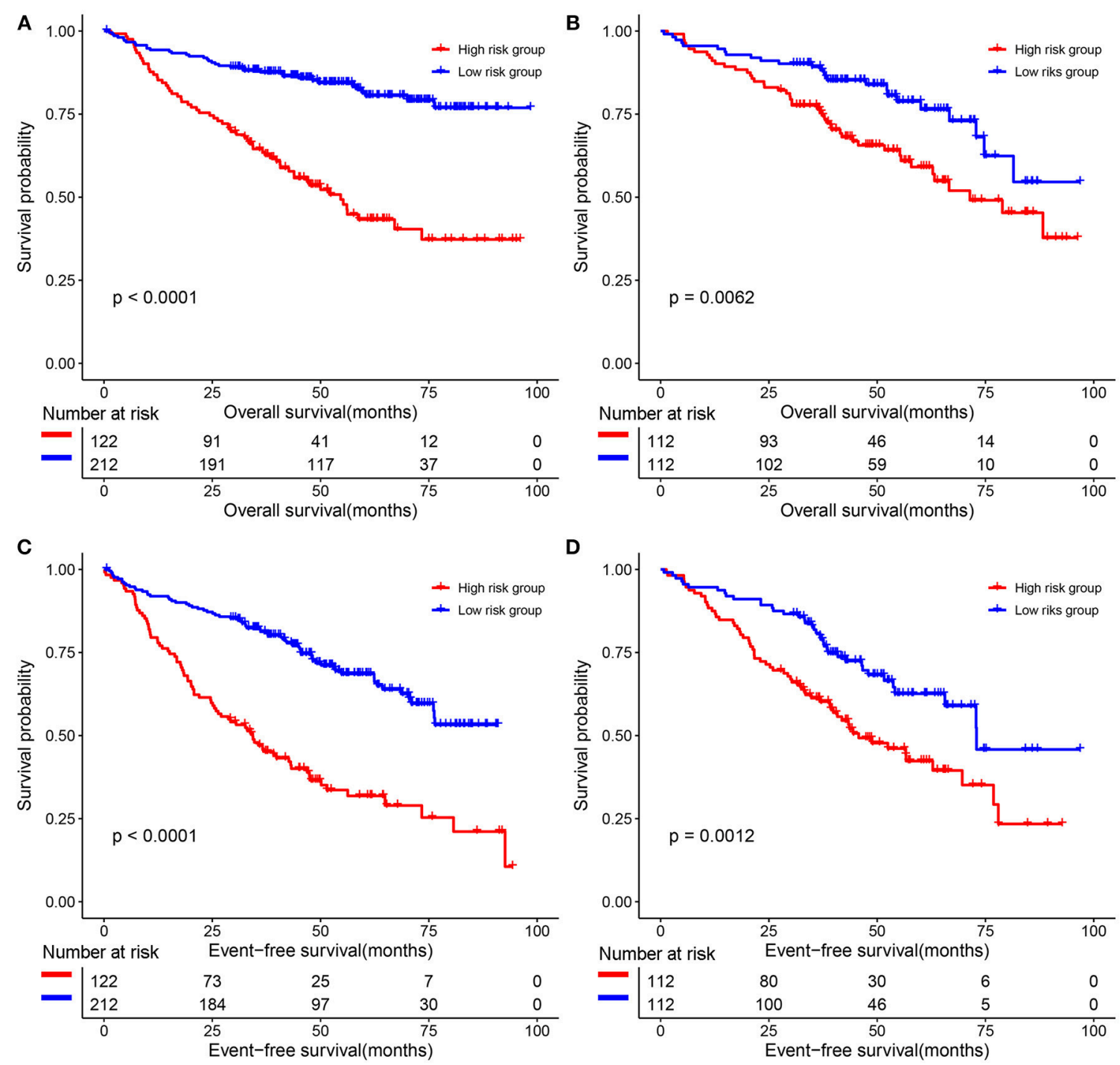

FIGURE 3 | The correlations between the 9-gene signature and the overall survival (OS) and event-free survival (EFS) of patients with MM. (A) OS in the training set. (B) OS in the test set. (C) EFS in the training set. (D) EFS in the test set. 


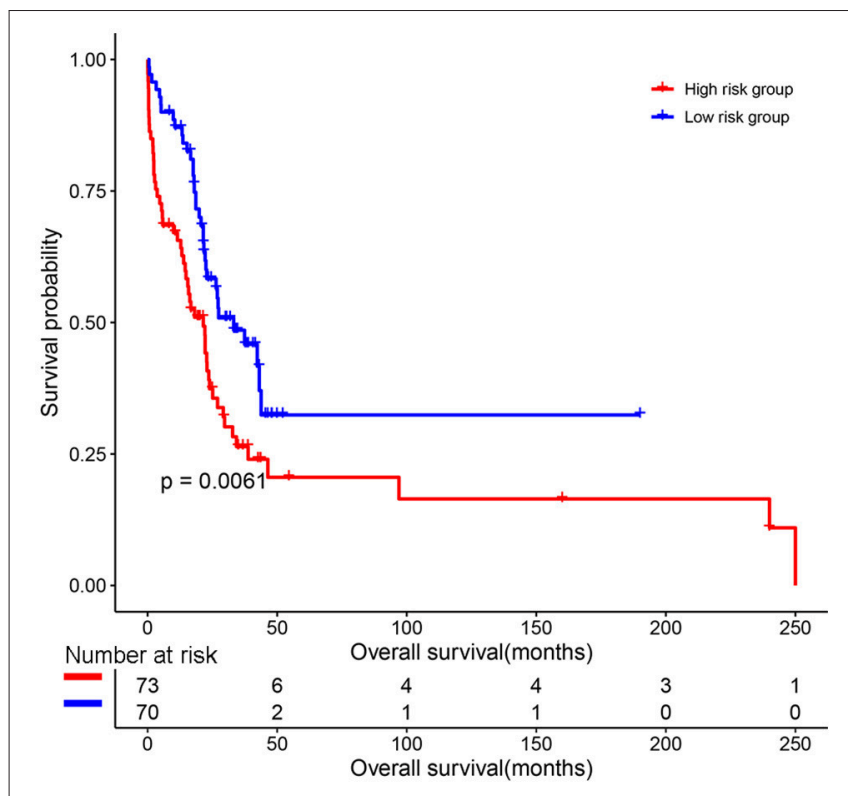

FIGURE 4 | The prognostic role of the 9-gene signature in the independent validation cohort E-MTAB-4032.

Cox proportional hazards suggested that the 9-gene signature was also an independent prognostic factor in the validation cohort $(\mathrm{HR}=14.8092,95 \%$ CI:1.2282-178.5591, $P=0.0339$, Supplementary Table 7).

\section{DISCUSSION}

As stated above, although several novel agents for patients with MM have been introduced into clinical practice, the disease remained incurable, and the clinical outcome of patients with MM is still poor $(2,3)$. Thus, it is of vital importance to develop such molecular biomarkers or signature that are significantly correlated with the clinicopathological features and clinical outcome of patients with MM. In the present study, a total of 758 hub genes associated with the ISS stage of MM patients were identified through WGCNA. Thus, we performed univariate Cox proportional hazards regression analysis to analyzed the relations between the expression of hub genes and the OS of patients with MM, and the results suggested that 325 hub genes were associated with the OS of MM patients. LASSO (34) was introduced in order to improve the prediction accuracy and interpretability of regression models by forcing the sum of the absolute value of the regression coefficients to be less than a fixed value, which forces certain coefficients to be set to zero, effectively choosing a simpler model that does not include those coefficients. Based on this, we included the above 325 hub genes into a LASSO penalized Cox proportional hazards regression model, as a result, 9 hub genes with none-zero coefficients in this model was identified. Thus, we calculated the risk score of each MM patients, and built the 9 hub genes [HLADPB1(major histocompatibility complex, class II, DP beta 1),
TOP2A (topoisomerase 2A), FABP5 (Fatty Acid-Binding Protein 5), CYP1B1(cytochrome P450 family 1 subfamily B member 1), IGHM (immunoglobulin heavy constant mu), FANCI (FA complementation group I), LYZ (lysozyme), HMGN5 (high mobility group protein N5 subtype), and BEND6 (BEN domain containing 6)] based signature.

Results of functional enrichment analysis of hub genes suggested that hub genes were mainly enriched in cell proliferation and immune response related GO terms and pathways, this was in accordance with the prognostic value of the 9-gene signature developed based on the hub genes. MM patients in the 9-gene low risk group was associated with better OS and EFS compared with those in the 9gene high risk group, and the 9-gene signature was shown to be an independent prognostic signature in patients with MM.

Actually, most of these hub genes in the signature had been demonstrated be associated with the proliferation or invasion of several human cancers. HLA-DPB1, also known as major histocompatibility complex, class II, DP beta 1 , belongs to the HLA-class IIbeta chain paralogue. Li et al. demonstrated that genetic variant of HLA-DPB1 increased in the risk of extranodal natural killer T-cell lymphoma (37). Liu et al. demonstrated that TOP2A (topoisomerase 2A) and TOP1 functioned as oncogene in liver cancer (38). Meanwhile, decreased expression of TOP2A inhibited the proliferation of invasion of colon cancer cells (39). Kawaguchi et al and Powell et al. demonstrated that overall expression of FABP5 promoted the proliferation and metastasis of colorectal cancer cells and breast cancer cells (40). Gu et al. demonstrated that genetic variant of CYP1B1 gene was associated prognosis of patients with prostate cancer (41). Mutation patterns of IGHM was associated different progression pathways in follicular lymphoma (42). Chen et al demonstrated that PRC1 could promote early recurrence of patients with hepatocellular carcinoma by regulating the expression of FANCI (43). Mariano FV et al demonstrated that the expression of LYZ could be used to differentiate mammary analog secretory carcinoma from acinic cell carcinoma of salivary glands (44). Wu et al demonstrated that the expression of HMGN5 was increased in bladder cancer cells and high expression of HMGN5 was associated with poor prognosis of patients with bladder cancer (45). The results of the above literature review provided strong support for the 9gene signature in the clinical out prediction in patients with MM.

Limits of our study are as follows. First, our study is a retrospective analysis based on previously published $\mathrm{MM}$ gene expression studies, although its conclusions have been confirmed in the test set and independent validation set, we recommend that the conclusions of this study should be verified by molecular biology experiments in subsequent studies. Second, the prognostic performance of the 9-gene signature should be evaluated through prospective clinical trials.

Taken together, we developed a 9-gene prognostic signature based on the hub genes obtained through a co-expression 
network, patients in the 9-gene signature low risk group were associated with better clinical outcomes compared with those in the 9-gene signature high risk group.

\section{AUTHOR CONTRIBUTIONS}

X-PL collected and analyzed the data, write the manuscript. XiaHY analyzed the data, and review the manuscript. X-YM and Xin-HY participated in revising the manuscript. FW revised the manuscript, analyzed the data, and participated in preparation of the figures. LH designed the study and participated in data analysis.

\section{FUNDING}

The Research Fund from the National Natural Science Foundation of China (No. 81800206).

\section{SUPPLEMENTARY MATERIAL}

The Supplementary Material for this article can be found online at: https://www.frontiersin.org/articles/10.3389/fonc. 2018.00615/full\#supplementary-material

\section{REFERENCES}

1. Merz M, Kellermann L, Poenisch W, Tischler HJ, Kohnke J, Knauf W, et al. Diagnosis and treatment of multiple myeloma in Germany: analysis of a nationwide multi-institutional survey. Ann Hematol. (2017) 96:987-93. doi: 10.1007/s00277-017-2991-0

2. Gozzetti A, Papini G, Defina M, Bocchia M. Extramedullary myeloma relapses. Ann Hematol. (2012) 91:1511-2. doi: 10.1007/s00277-012-1432-3

3. Chan HSH, Chen CI, Reece DE. Current review on high-risk multiple myeloma. Curr Hematol Malig Rep. (2017) 12:96-108. doi: 10.1007/s11899-017-0368-z

4. Rajkumar SV, Buadi F. Multiple myeloma: new staging systems for diagnosis, prognosis and response evaluation. Best Pract Res Clin Haematol. (2007) 20:665-80. doi: 10.1016/j.beha.2007.10.002

5. Bataille R, Annweiler C, Beauchet O. Multiple myeloma international staging system: "staging" or simply "aging" system? Clin Lymphoma Myeloma Leuk (2013) 13:635-7. doi: 10.1016/j.clml.2013.07.003

6. MacLennan IC, Drayson M, Dunn J. Multiple myeloma. BMJ (1994) 308:1033-6. doi: 10.1136/bmj.308.6935.1033

7. Bergsagel PL. Where we were, where we are, where we are going: progress in multiple myeloma. Am Soc Clin Oncol Educ Book (2014) 2014:199-203. doi: 10.14694/EdBook_AM.2014.34.199

8. Bergin K, McQuilten Z, Moore E, Wood E, Spencer A. Myeloma in the real world: what is really happening? Clin Lymphoma Myeloma Leuk (2017) 17:133-144 e131. doi: 10.1016/j.clml.2016.12.002

9. Liu X, Chen J, He YA, Meng X, Li K, He CK, et al. Comparing efficacy and survivals of initial treatments for elderly patients with newly diagnosed multiple myeloma: a network meta-analysis of randomized controlled trials. Onco Targets Ther. (2017) 10:121-8. doi: 10.2147/OTT.S123680

10. Berenson A, Vardanyan S, David M, Wang J, Harutyunyan NM, Gottlieb J, et al. Outcomes of multiple myeloma patients receiving bortezomib, lenalidomide, and carfilzomib. Ann Hematol. (2017) 96:449-59. doi: 10.1007/s00277-016-2889-2

11. Terpos E, International Myeloma S. Multiple myeloma: clinical updates from the american society of hematology annual meeting 2016. Clin Lymphoma Myeloma Leuk (2017) 17:329-39. doi: 10.1016/j.clml.2017.02.010
Supplementary Figure 1 | Flowchart depicting the major process of Co-expression network construction and identification of hub genes.

Supplementary Figure 2 | Time-Dependent ROC Curve for the 9-gene signature in the training set at 90 months.

Supplementary Table 1 | Hub genes calculated using weighted co- expression network analysis and their impact on the overall survival of patients with multiple myeloma.

Supplementary Table 2 | The 9 hub genes with non-zero coefficients in the LASSO Cox proportional hazards regression model.

Supplementary Table 3 | Cox proportional hazards regression analysis the association between the clinical characteristics and overall survival of multiple myeloma in the training set.

Supplementary Table 4 | Cox proportional hazards regression analysis on the association between the clinical characteristics and overall survival of multiple myeloma patients in the test. set.

Supplementary Table 5 | Cox proportional hazards regression analysis on the association between the clinical characteristics and event-free survival of multiple myeloma patients in the training set.

Supplementary Table 6 | Cox proportional hazards regression analysis on the association between the clinical characteristics and event-free survival of multiple myeloma patients in the test set.

Supplementary Table 7 | The prognostic role of the 9-gene signature in the independent validation cohort.

12. Cejalvo MJ, de la Rubia J. Which therapies will move to the front line for multiple myeloma? Exp Rev Hematol. (2017) 10:383-92. doi: 10.1080/17474086.2017.1317589

13. Cejalvo MJ, de la Rubia J. Clinical treatment of newly diagnosed multiple myeloma. Expert Rev Hematol. (2015) 8:595-611. doi: $10.1586 / 17474086.2015 .1078236$

14. Greipp PR, San Miguel J, Durie BG, Crowley JJ, Barlogie B, Blade J, et al. International staging system for multiple myeloma. J Clin Oncol. (2005) 23:3412-20. doi: 10.1200/JCO.2005.04.242

15. Kyrtsonis MC, Maltezas D, Tzenou T, Koulieris E, Bradwell AR. Staging systems and prognostic factors as a guide to therapeutic decisions in multiple myeloma. Semin Hematol. (2009) 46:110-7. doi: 10.1053/j.seminhematol.2009.02.004

16. Zhang B, Horvath S. A general framework for weighted gene coexpression network analysis. Stat Appl Genet Mol Biol. (2005) 4:17. doi: $10.2202 / 1544-6115.1128$

17. Yip AM, Horvath S. Gene network interconnectedness and the generalized topological overlap measure. BMC Bioinformatics (2007) 8:22. doi: $10.1186 / 1471-2105-8-22$

18. Li S, Liu X, Liu T, Meng X, Yin X, Fang C, et al. Identification of Biomarkers Correlated with the TNM Staging and Overall Survival of Patients with Bladder Cancer. Front Physiol. (2017) 8:947. doi: 10.3389/fphys.2017.00947

19. Broyl A, Hose D, Lokhorst H, de Knegt Y, Peeters J, Jauch A, et al. Gene expression profiling for molecular classification of multiple myeloma in newly diagnosed patients. Blood (2010) 116:2543-53. doi: 10.1182/blood-2009-12-261032

20. Shi L, Campbell G, Jones WD, Campagne F, Wen Z, Walker SJ, et al. The MicroArray Quality Control (MAQC)-II study of common practices for the development and validation of microarray-based predictive models. Nat Biotechnol .(2010) 28:827-38. doi: 10.1038/nbt.1665

21. Chng WJ, Kumar S, Vanwier S, Ahmann G, Price-Troska T, Henderson K, et al. Molecular dissection of hyperdiploid multiple myeloma by gene expression profiling. Cancer Res. (2007) 67:2982-9. doi: 10.1158/0008-5472.CAN-06-4046

22. Tiedemann RE, Zhu YX, Schmidt J, Yin H, Shi CX, Que Q, et al. Kinome-wide RNAi studies in human multiple myeloma identify vulnerable kinase targets, 
including a lymphoid-restricted kinase, GRK6. Blood (2010) 115:1594-604. doi: 10.1182/blood-2009-09-243980

23. Kryukov F, Nemec P, Radova L, Kryukova E, Okubote S, Minarik J, et al. Centrosome associated genes pattern for risk sub-stratification in multiple myeloma. J Transl Med. (2016) 14:150. doi: 10.1186/s12967-016-0906-9

24. Gautier L, Cope L, Bolstad BM, Irizarry RA. affy-analysis of Affymetrix GeneChip data at the probe level. Bioinformatics (2004) 20:307-15. doi: 10.1093/bioinformatics/btg405

25. Carvalho BS, Irizarry RA. A framework for oligonucleotide microarray preprocessing. Bioinformatics (2010) 26:2363-7. doi: 10.1093/bioinformatics/btq431

26. Irizarry RA, Hobbs B, Collin F, Beazer-Barclay YD, Antonellis KJ, Scherf U, et al. Exploration, normalization, and summaries of high density oligonucleotide array probe level data. Biostatistics (2003) 4:249-64. doi: 10.1093/biostatistics/4.2.249

27. Langfelder $\mathrm{P}$, Horvath S. WGCNA: an $\mathrm{R}$ package for weighted correlation network analysis. BMC Bioinformatics (2008) 9:559. doi: 10.1186/1471-2105-9-559

28. Oldham MC, Langfelder P, Horvath S. Network methods for describing sample relationships in genomic datasets: application to Huntington's disease. BMC Syst Biol. (2012) 6:63. doi: 10.1186/1752-0509-6-63

29. Li A, Horvath S. Network module detection: Affinity search technique with the multi-node topological overlap measure. BMC Res Notes (2009) 2:142. doi: 10.1186/1756-0500-2-142

30. Dong J, Horvath S. Understanding network concepts in modules. BMC Syst Biol. (2007) 1:24. doi: 10.1186/1752-0509-1-24

31. Huang da W, Sherman BT, Lempicki RA. Systematic and integrative analysis of large gene lists using DAVID bioinformatics resources. Nat Protoc. (2009) 4:44-57. doi: 10.1038/nprot.2008.211

32. Ito K, Murphy D. Application of ggplot2 to Pharmacometric Graphics. CPT Pharmacometr Syst Pharmacol. (2013) 2:e79. doi: 10.1038/psp.2013.56

33. Wang H, Lengerich BJ, Aragam B, Xing EP. Precision lasso: accounting for correlations and linear dependencies in high-dimensional genomic data. Bioinformatics (2018). doi: 10.1093/bioinformatics/bty750. [Epub ahead of print].

34. Friedman J, Hastie T, Tibshirani R. Regularization paths for generalized linear models via coordinate descent. J Stat Softw. (2010) 33:1-22. doi: $10.18637 /$ jss.v033.i01

35. Holleczek B, Brenner H. Model based period analysis of absolute and relative survival with $\mathrm{R}$ : data preparation, model fitting and derivation of survival estimates. Comput Methods Programs Biomed. (2013) 110:192-202. doi: 10.1016/j.cmpb.2012.10.004

36. Lorent M, Giral M, Foucher Y. Net time-dependent ROC curves: a solution for evaluating the accuracy of a marker to predict disease-related mortality. Stat Med. (2014) 33:2379-89. doi: 10.1002/sim.6079
37. Li Z, Xia Y, Feng LN, Chen JR, Li HM, Cui J, et al. Genetic risk of extranodal natural killer T-cell lymphoma: a genome-wide association study. Lancet Oncol. (2016) 17:1240-7. doi: 10.1016/S1470-2045(16)30148-6

38. Liu LM, Xiong DD, Lin P, Yang H, Dang YW, Chen G. DNA topoisomerase 1 and $2 \mathrm{~A}$ function as oncogenes in liver cancer and may be direct targets of nitidine chloride. Int J Oncol. (2018) 53:1897-912. doi: 10.3892/ijo.2018.4531

39. Zhang R, Xu J, Zhao J, Bai JH. Proliferation and invasion of colon cancer cells are suppressed by knockdown of TOP2A. J Cell Biochem. (2018) 119:7256-63. doi: $10.1002 /$ jcb. 26916

40. Kawaguchi K, Senga S, Kubota C, Kawamura Y, Ke Y, Fujii H. High expression of Fatty Acid-Binding Protein 5 promotes cell growth and metastatic potential of colorectal cancer cells. FEBS Open Bio (2016) 6:190-9. doi: 10.1002/2211-5463.12031

41. Gu CY, Qin XJ, Qu YY, Zhu Y, Wan FN, Zhang GM, et al. Genetic variants of the CYP1B1 gene as predictors of biochemical recurrence after radical prostatectomy in localized prostate cancer patients. Medicine (2016) 95:e4066. doi: 10.1097/MD.0000000000004066

42. Ruminy P, Jardin F, Picquenot JM, Parmentier F, Contentin N, Buchonnet $\mathrm{G}$, et al. S(mu) mutation patterns suggest different progression pathways in follicular lymphoma: early direct or late from FL progenitor cells. Blood (2008) 112:1951-9. doi: 10.1182/blood-2007-11-124560

43. Chen J, Rajasekaran M, Xia H, Zhang X, Kong SN, Sekar K, et al. The microtubule-associated protein $\mathrm{PRCl}$ promotes early recurrence of hepatocellular carcinoma in association with the Wnt/beta-catenin signalling pathway. Gut (2016) 65:1522-34. doi: 10.1136/gutjnl-2015-3 10625

44. Mariano FV, Gomez CA, de Souza do Nascimento J, Dos Santos HT, Egal ES, Montalli, VA, et al. Lysozyme expression can be useful to distinguish mammary analog secretory carcinoma from acinic cell carcinoma of salivary glands. Head Neck Pathol. (2016) 10:429-36. doi: 10.1007/s12105-016-0718-5

45. Wu J, Wang J. HMGN5 expression in bladder cancer tissue and its role on prognosis. Eur Rev Med Pharmacol Sci. (2018) 22:970-5. doi: 10.26355/eurrev_201802_14378

Conflict of Interest Statement: The authors declare that the research was conducted in the absence of any commercial or financial relationships that could be construed as a potential conflict of interest.

Copyright $\odot 2019 \mathrm{Liu}$, Yin, Meng, Yan, Wang and He. This is an open-access article distributed under the terms of the Creative Commons Attribution License (CC BY).

The use, distribution or reproduction in other forums is permitted, provided the original author(s) and the copyright owner(s) are credited and that the original publication in this journal is cited, in accordance with accepted academic practice. No use, distribution or reproduction is permitted which does not comply with these terms. 